Trinity University

Digital Commons@ Trinity

Engineering Faculty Research

Engineering Science Department

$10-2013$

\title{
Vapor Distribution above an Evaporating Sessile Drop
}

Peter Kelly-Zion

TrinityUniversity, plkelly@trinity.edu

Christopher J. Pursell

TrinityUniversity, cpursell@trinity.edu

N Hasbamrer

Trinity University

B Cardozo

Trinity University

K Gaughan

Trinity University

See next page for additional authors

Follow this and additional works at: https://digitalcommons.trinity.edu/engine_faculty

Part of the Engineering Commons

\section{Repository Citation}

P.L. Kelly-Zion, C.J. Pursell, N. Hasbamrer, B. Cardozo, K. Gaughan, K. Nickels, Vapor distribution above an evaporating sessile drop, International Journal of Heat and Mass Transfer, Volume 65, October 2013, Pages 165-172, ISSN 0017-9310, http://dx.doi.org/ 10.1016/j.ijheatmasstransfer.2013.06.003.

This Post-Print is brought to you for free and open access by the Engineering Science Department at Digital Commons @ Trinity. It has been accepted for inclusion in Engineering Faculty Research by an authorized administrator of Digital Commons @ Trinity. For more information, please contact jcostanz@trinity.edu. 
Authors

Peter Kelly-Zion, Christopher J. Pursell, N Hasbamrer, B Cardozo, K Gaughan, and Kevin Nickels 


\title{
Vapor Distribution above an Evaporating Sessile Drop
}

\author{
P.L. Kelly-Zion ${ }^{\mathrm{a}, *}$, C.J. Pursell ${ }^{\mathrm{b}}$, N. Hasbamrer ${ }^{\mathrm{a}}$, B. Cardozo ${ }^{\mathrm{a}}$, K. Gaughan ${ }^{\mathrm{a}}$, and K. Nickels ${ }^{\mathrm{a}}$ \\ ${ }^{a}$ Department of Engineering Science \\ Trinity University, San Antonio 78212, United States \\ ${ }^{\mathrm{b}}$ Department of Chemistry \\ Trinity University, San Antonio 78212, United States
}

\begin{abstract}
An experimental technique was developed that uses infrared tomography to measure the three-dimensional vapor distribution above an evaporating sessile drop. The technique was applied to measure the vapor distributions above evaporating drops of hexane and 3-methylpentane (3MP) at room temperature and pressure. The molecular masses of these two species are heavier than air and the vapor from the evaporating drop forms a flat, disk-shaped cloud. A Fourier transform infrared spectrometer (FTIR) was used to measure the spectral absorbance along a set of paths passing through the vapor cloud. From a set of pathaveraged absorbance measurements, a two-dimensional spatial concentration distribution was determined using a computed tomography routine. A three-dimensional concentration distribution was obtained from multiple two-dimensional distributions obtained at different elevations above the drop.

The vapor distributions for both hexane and 3MP differ significantly from the values predicted by the solutions for diffusionlimited evaporation and indicate the effect of buoyancy-induced convection of the vapor. These measurements are the first quantitative measurements of the vapor distribution above a sessile drop and are important for advancing the understanding of the vapor phase transport mechanisms, and thus sessile drop evaporation.
\end{abstract}

\section{Keywords}

Evaporation; drop; vapor distribution; convection; diffusion

\section{Nomenclature}

A absorbance

l path length through vapor

C molar concentration

$r \quad$ radial coordinate

$x \quad$ horizontal coordinate

$z \quad$ vertical coordinate

Greek Symbols

$\alpha \quad$ molecular absorptivity

$v \quad$ spatial frequency

\section{Subscripts}

$v \quad$ spectral quantity

sat saturation value

\section{Introduction}

The understanding of sessile drop evaporation is important for many technical applications, including spray cooling [1,2], coating [3], ink-jet printing [4-6], DNA stretching and depositing [7,8], and self-assembly and surface patterning[9-11]. Analytical expressions for drop evaporation of various complexities have been developed for quasi-steady evaporation when the rate is limited by the rate of vapor diffusion from the drop surface[12-17]. Erbil has written a comprehensive review of the analytical models that have been developed over the past 120 years [18]. In addition, computational models have been developed to include complex phenomena not accounted for by the analytical models. For example, Dunn et al. developed a computational model to account for evaporative cooling and the pressure dependence of the vapor diffusivity [19], Semenov et al. computed the instantaneous flux distributions [20], and Widjaja and Harris studied the effects of motion inside the liquid drop [21].

The great majority of the published research on sessile drop evaporation pertains to conditions of quasi-steady, vapor diffusionlimited evaporation. Less work has been published for conditions in which buoyancy-induced convection of the vapor is

* Corresponding author: Tel.: 1210999 7518; fax: 12109998037

E-mail address: peter.kelly-zion@trinity.edu (P.L. Kelly-Zion) 
significant. Recently, researchers have included vapor-phase natural convection in their models for the evaporation of sessile water drops [22,23]. Saada et al. computed a convective velocity distribution that is radially inward above the perimeter of the drop and turns vertically upward above the center of the drop due to the water vapor being less dense than the surrounding air. That result is qualitatively similar to the conclusions of O’Brien and Saville, who used interferometry to obtain images of the vapor above a diethyl ether drop and stated that the images are consistent with the presence of Bénard cell circulation with the vapor flow directed up along an axis above the center of the drop [24]. For the case of vapor that is heavier than air, schlieren videos show the convective flow of the vapor-air mixture to be horizontal and directed away from the drop surface in all radial directions [25]. Carle et al. investigated the effect of natural convection of vapor on the evaporation rates of ethanol drops by comparing the rates under conditions of normal and microgravity [26], and Vynnycky and Maeno considered the case of the water drop being colder than the surrounding air, and thus the water vapor being heavier than the air, but the results for the velocity and concentration distributions of the vapor were not presented [23].

Very little information is published on the distribution of the vapor concentration surrounding a sessile drop. For the case of diffusion-limited evaporation, the vapor is generally assumed to be distributed in the approximately hemispherical space above the drop according to the solution of the steady Laplace equation [15,16,19,21,27]. A computational model that includes buoyancy-induced convection in the vapor phase for water vapor (lighter than air) indicates that convection draws the vapor toward a vertical axis above the center of the drop so that lines of constant concentration appear cone-shaped as opposed to hemispherical [22]. When the vapor is heavier than air, the vapor is contained in a flat, disk-shaped cloud surrounding the drop [25].

The distribution of the vapor is an important characteristic of the evaporation process. The local rate of diffusion is determined by the concentration gradient, and buoyancy-induced convection is determined by the density gradient. Since both convection and diffusion influence the vapor distribution, the two mechanisms are coupled so that the occurrence of convection affects the rate of diffusion, and vice versa. Thus, quantitative knowledge of the vapor distribution can lead to a more thorough understanding of the vapor transport. Furthermore, the conditions at the surface of the drop can influence the liquid phase behavior. For example, a shear stress induced by a convective flow of the vapor-air mixture at the surface of the drop will cause motion within the liquid drop. As mentioned above, the effects of various physical processes on the evaporation of sessile drops have been modelled, but there have not been any quantitative measurements of the vapor distribution, which are important for determining whether the models are accurately computing the vapor transport when both diffusion and convection are significant.

This paper presents a technique for obtaining quantitative measurements of the vapor distribution above a sessile drop using infrared spectroscopy and computed tomography. Computed tomography is a procedure to obtain a two-dimensional distribution (slice) from a series of one-dimensional measurements. By combining slices, a three-dimensional distribution can be obtained. The use of computed tomography is well known in medical applications, i.e. CT or CAT scans, but also it has been used in a variety of research applications, for example measuring combustion products in an internal combustion engine [28] or from a flat flame burner [29], measuring the structure of a hollow cone fuel spray [30], measuring nitrogen emissions from a swine waste lagoon [31], and various non-destructive testing applications [32].

We used computed tomography to determine the three-dimensional distributions of vapor concentration above sessile drops of hexane and 3-methylpentane (3MP) from a series of infrared absorbance measurements obtained using a Fourier transform infrared spectrometer (FTIR). A significant advantage of using an FTIR over a monochromatic source is the potential to simultaneously measure the vapor concentrations of different species, as occurs during the evaporation of multicomponent drops. That potential will be investigated in a future paper; for this paper the technique is applied to measure the vapor concentration surrounding single-component drops.

The measured vapor distributions for both hexane and 3MP are much different than the distributions that would be expected for diffusion-limited evaporation, and thus the measurements indicate the effect of buoyancy-induced convection.

\section{Method}

Figure 1 is a schlieren image of the vapor cloud surrounding a pinned, sessile hexane drop of radius $6.5 \mathrm{~mm}$ and having an initial volume of $80 \mu \mathrm{l}$. The bright region indicates the presence of vapor. The drop is located at the center of the disk-shaped substrate, which has a radius of $22 \mathrm{~mm}$. Since the vapor is heavier than air, the cloud flows away from the drop along the surface of the substrate and spills over the edge. As a result, the shape of the cloud is relatively flat with a thickness of approximately $5 \mathrm{~mm}$.

To measure the vapor concentration, the infrared (IR) beam of an FTIR is passed horizontally through the vapor cloud at various locations above the evaporating drop. The arrow in Fig. 1 represents one pass of the IR beam. For each pass, the FTIR measures the absorption of the vapor-air mixture in the path of the beam, which is proportional to the average concentration along the beam path. To determine the two-dimensional vapor distribution in a planar slice through the cloud, multiple path-averaged absorption measurements are needed. For that purpose, a series of measurements are taken with the infrared beam passing through different secants of the vapor cloud in a given elevation plane, as shown in Fig. 2a. Constrained by the width of the sample compartment of the FTIR, not all regions of the cloud were probed but measurements were taken over more than half of the planar slice and extended beyond the edge of the cloud. 


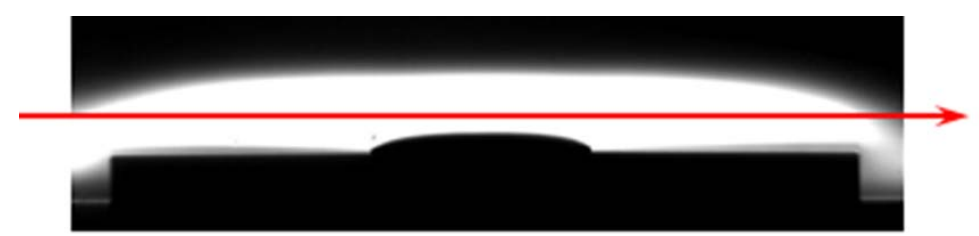

Figure 1. Schlieren image of the vapor cloud surrounding a pinned, sessile hexane drop. The bright region indicates the presence of vapor and the arrow demonstrates a path of the infrared beam from the FTIR through the vapor cloud.

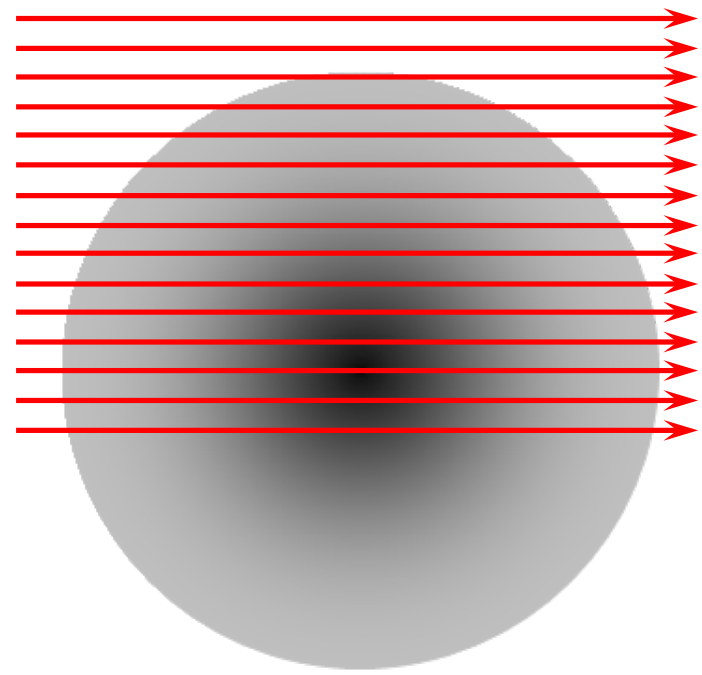

2a. Series of IR absorption measurements.

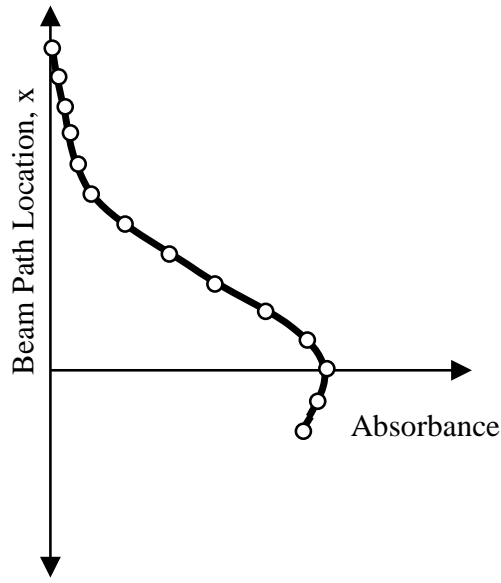

2b. Projection of the two-dimensional absorbance distribution.

Figure 2. Schematic of a top view of the vapor cloud (grey region) and a series of IR absorption measurements made in a single elevation plane above the drop (Fig. 2a). The resulting distribution of the IR absorption measurements, which is equivalent to a one-dimensional projection of the two-dimensional absorbance distribution, is shown in Fig. $2 \mathrm{~b}$.

The distribution of path-averaged absorbance values, shown schematically in Fig. 2b, is the one-dimensional projection of the two-dimensional absorbance distribution in the given plane of the vapor cloud. To compute the vapor concentration distribution in an elevation plane, axial symmetry is assumed and computed tomography (CT) is used to compute the two-dimensional absorbance distribution from the measured one-dimensional projection. The vapor distribution, then, is obtained by multiplying the absorbance distribution by the molar absorptivity, which is measured by a separate series of experiments. Combining the distributions in multiple elevation planes results in the three-dimensional vapor distribution.

There are multiple algorithms for computing tomographic reconstructions from parallel beam measurements. The algorithm used for this work is Filtered Back Projection (FBP), which is the most used algorithm for straight ray tomography [33]. FBP makes use of the Fourier Slice Theorem to compute the two-dimensional absorption distribution based on the one-dimensional projection.

By assuming the vapor distribution is axially symmetric, the measured projection, which is acquired at one angle, can be used to generate an equivalent projection for any angle. The resolution of the resulting distribution increases with the number of angles at which projections are taken. Following the guidance of Kak and Slaney [34] the number of angles used for each elevation plane was equal to the number of discrete values in the projection. Typically, this number was 120.

Computations were accomplished using Matlab ${ }^{\circledR}$ [35]. Matlab’s internal function 'iradon’ was used to perform the inverse Radon transform in order to generate the two-dimensional absorbance distribution.

Since the vapor distribution is unknown, the accuracy of the CT routine was tested by mathematically generating a distribution to simulate the vapor cloud. The absorbance values of lines passing through different locations of the simulated cloud were then computed to act as the IR absorption measurements. These "measurements" were inputted in the CT routine and the output was compared to the known distribution. This test was repeated for a variety of distributions and in all cases the output of the CT routine closely matched the known distribution.

The details of the experimental methodology are discussed in the following three subsections. First, the experimental technique for obtaining the absorption measurements using the FTIR is discussed, followed by descriptions of the procedures for analyzing the absorption data and generating the input data for the CT analysis. A third subsection discusses how the results of the CT analysis were processed to yield the three-dimensional vapor concentration distribution. 


\subsection{FTIR Absorption Measurements}

A sessile drop was generated by injecting approximately $80 \mu \mathrm{L}$ of hexane or 3MP onto a $6.5 \mathrm{~mm}$ radius disk which is embedded at the center of a copper substrate of radius $22 \mathrm{~mm}$ as shown in Fig. 3. The central disk is raised slightly $(0.2 \mathrm{~mm})$ above the surface of the copper substrate to provide a circular edge along the perimeter of the disk to which the injected liquid attaches by the effect of surface tension. In this way, the drop, which covers the surface of the disk, is pinned so that the radius of the contact line remains constant for the majority of the drop's lifetime. Eventually, as the drop evaporates, insufficient liquid remains to cover the surface of the disk and the drop pulls from the edge. Only measurements taken during the time a drop remains fully attached are used in the analyses.

The initial contact angle for the hexane drop was measured, in a different experiment under analogous conditions, to be $28^{\circ}$ and the angle reduced at a constant rate of $0.2 \mathrm{deg} / \mathrm{s}$. Although the contact angle of the 3MP drop was not measured, it appeared to be equal to that of the hexane drop.

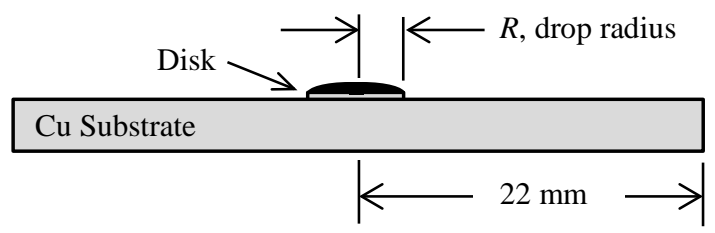

Figure 3. Schematic of a profile view of a sessile drop (black) which covers the surface of a disk at the center of the copper substrate. The drop is attached around the circular periphery of the disk and in this way the drop size is maintained during the majority of the evaporation process.

The substrate was affixed to an $\mathrm{x}-\mathrm{z}$ translation stage which was contained in the sample compartment of a Nicolet 550 FTIR. The translation stage enabled the precise positioning $( \pm 0.1 \mathrm{~mm})$ of the substrate in two directions, horizontally normal to the IR beam and vertically below the IR beam. The volume of the FTIR's sample compartment is $10,400 \mathrm{~cm}^{3}$, which is sufficiently large to ensure that the vapor mole fraction was never more than $0.14 \%$. During an experiment, the sample compartment was closed to prevent drafts from affecting the vapor distribution. The experiments were conducted at a temperature of $22.6 \pm 0.5^{\circ} \mathrm{C}$ and an ambient pressure of $96 \pm 2 \mathrm{kPa}$.

To measure the average absorption along a specified path passing through the vapor cloud, the position of the substrate relative to the IR beam was set using the translation stage. The position of the beam path relative to the drop was specified using a coordinate system with the origin located at the center of the surface of the central disk. The coordinates (x, z) give the horizontal and vertical position of the beam, with the beam always oriented normal to the $\mathrm{x}-\mathrm{z}$ plane. The $\mathrm{x}$ coordinate is the horizontal distance to the location where the beam intersects the $\mathrm{x}-\mathrm{z}$ plane, as depicted in Fig. 4.

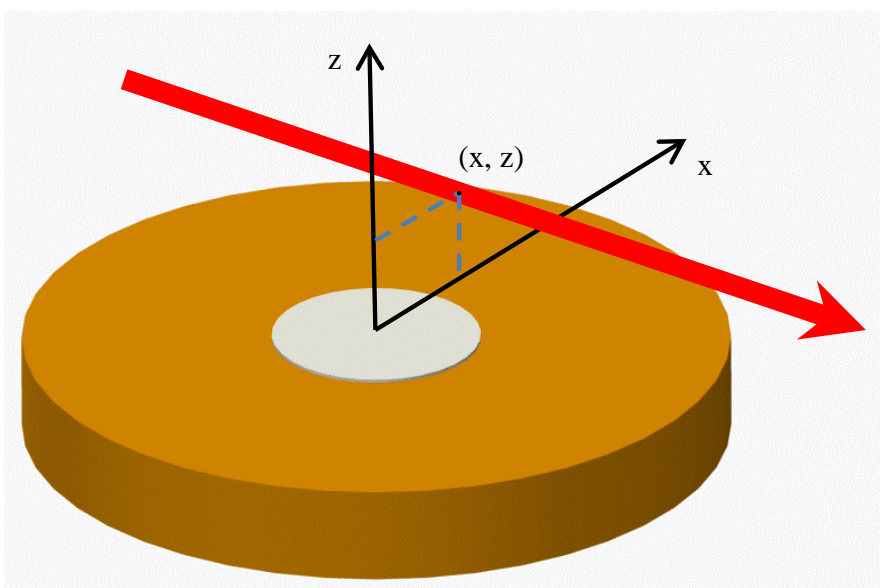

Figure 4. Schematic of the coordinate system centered on the top surface of the central disk. The IR beam was oriented normal to the $\mathrm{x}-\mathrm{z}$ plane.

The beam cross-section was oval shaped with a height of $3.1 \mathrm{~mm}$ and a width of $2.1 \mathrm{~mm}$, as defined by the locations where the beam intensity drops to $1 \%$ of the total intensity. The position of the beam path was specified at the geometric center of the beam’s cross section. However, as discussed in §2.3, the position of the resulting computed vapor distribution was adjusted in order to correspond to the location of maximum intensity within the beam, which occurred slightly above the geometric center.

A macro routine was used to automate the acquisition of the background interferogram and the subsequent absorbance spectra. The macro was initiated prior to the injection of the liquid drop in order to obtain a clean background interferogram and a set of 
absorbance spectra that spans the full lifetime of the drop. Due to the FTIR settings that were used, the time interval between absorbance spectra was approximately $6.15 \mathrm{~s}$. For each position, the measurement was repeated at least two times resulting in a minimum of three sets of transient absorbance data per position. The procedure was repeated for the other positions along the different secants through the vapor cloud, as shown schematically by the arrows in Fig. 2a, which are needed to compute the twodimensional vapor concentration distribution in the plane of the measurements.

Two typical absorbance spectra obtained during measurements of the hexane vapor concentration are presented in Fig. 5 . Both spectra were obtained for beams passing through the horizontal center of the cloud $(\mathrm{x}=0)$ but one spectrum was obtained at $\mathrm{z}=1$ $\mathrm{mm}$, where the average vapor concentration is high, and the other at $\mathrm{z}=3 \mathrm{~mm}$, where the average concentration is low. Each spectrum represents a measurement of the average vapor concentration along the path of the beam at one instant in time.

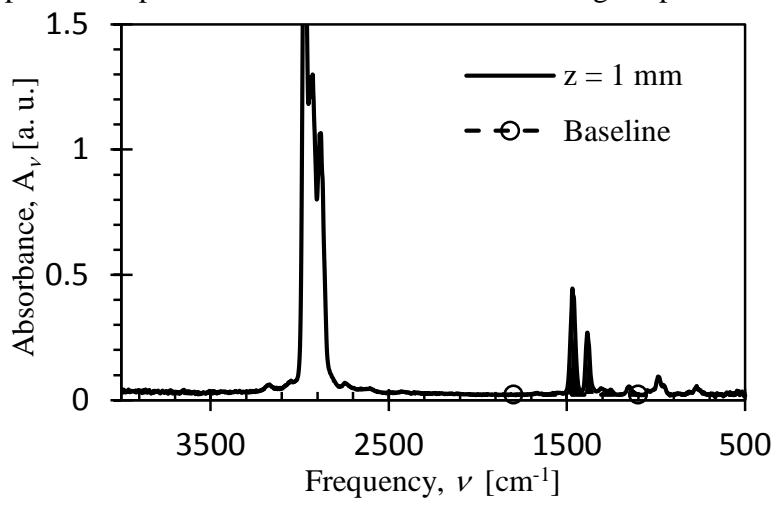

(a)

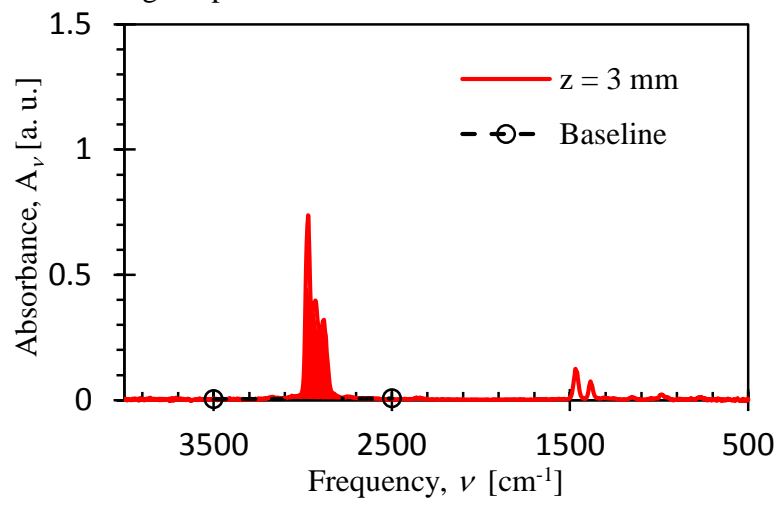

(b)

Figure 5. Two absorbance spectra representative of measurements taken at elevations of $1 \mathrm{~mm}$ (Fig. 5a) and $3 \mathrm{~mm}$ (Fig. 5b) for IR beams passing over the center of the hexane drop. Due to the high vapor concentration directly above the drop at $\mathrm{z}=1 \mathrm{~mm}$, the absorbance peak between $3100-2800 \mathrm{~cm}^{-1}$ was saturated and so the peak between $1520-1420 \mathrm{~cm}^{-1}$ was used. For elevations greater than $1 \mathrm{~mm}$, the absorbance peak between $3100-2800 \mathrm{~cm}^{-1}$ was used. The shaded regions represent the integrated absorbance values that were used.

Absorbance is related to concentration according to the Beer-Lambert Law, which equates spectral absorbance, $A_{v}$, to the product of the molar concentration, $C$, the molar absorptivity, $\alpha_{v}$, and the path length, $l$. To decrease sensitivity to experimental noise, the integrated absorbance of a specified spectral peak was used to determine the average concentration. The shaded regions in Fig. 5 indicate the integrated absorbances that were used. In general, the strongest peak, between 3100 and $2800 \mathrm{~cm}^{-1}$, was used. However, for measurements acquired close to the drop surface this peak was saturated and no longer followed the Beer-Lambert Law. At those measurement locations, the integrated absorbance between 1520 and $1420 \mathrm{~cm}^{-1}$ was used instead. To reduce errors associated with small fluctuations amongst the spectra, the common spectroscopic practice of defining a baseline was utilized. Integration was performed on the difference between the measured absorbance and the baseline, which was defined by fitting a line between the absorbance values at two frequencies where the vapor is transparent to IR light.

The molar absorptivities of the two spectral regions were measured by separate calibration experiments. A known amount of vapor was introduced into an evacuated gas cell and an FTIR was used to measure the absorbance spectra of the vapor. The vapor concentration was varied from zero to nearly the saturation value at room temperature, and the integrated absorbance was plotted versus concentration. The slope of the linear relationship between integrated absorbance and concentration is the product of integrated absorptivity and path length $(\alpha l)$. Thus, the integrated absorptivity was computed by dividing the slope by the path length through the gas cell. The absorptivity values for the two peaks for hexane and 3MP are given in Table 1 along with the integration limits and the two frequencies that define the baseline for each of the two peaks.

Table 1. Molar absorptivities, integration limits, and baseline definitions for hexane and 3MP.

\begin{tabular}{|c|c|c|c|c|}
\hline Species & $\begin{array}{c}\text { Peak 1: } \\
\text { Integration Limits } \\
\text { (Baseline Points) } \\
{\left[\mathrm{cm}^{-1}\right]} \\
\end{array}$ & $\begin{array}{c}\text { Peak 1: } \\
\alpha_{1} \\
\mathrm{~cm}^{-1} \\
\left(\mathrm{~mol} / \mathrm{cm}^{3}\right) \cdot \mathrm{mm} \\
\end{array}$ & $\begin{array}{c}\text { Peak 2: } \\
\text { Integration Limits } \\
\text { (Baseline Points) } \\
{\left[\mathrm{cm}^{-1}\right]} \\
\end{array}$ & $\begin{array}{c}\text { Peak 2: } \\
\alpha_{2} \\
\mathrm{~cm}^{-1} \\
\left(\mathrm{~mol} / \mathrm{cm}^{3}\right) \cdot \mathrm{mm} \\
\end{array}$ \\
\hline Hexane & $\begin{array}{c}3100-2800 \\
(3500-2500)\end{array}$ & $1.954 \times 10^{6}$ & $\begin{array}{c}1520-1420 \\
(1800-1100)\end{array}$ & $1.108 \times 10^{5}$ \\
\hline 3MP & $\begin{array}{c}3100-2800 \\
(3500-2500)\end{array}$ & $1.829 \times 10^{6}$ & $\begin{array}{c}1520-1420 \\
(1800-1100)\end{array}$ & $1.356 \times 10^{5}$ \\
\hline
\end{tabular}




\subsection{Analysis of Absorption Data and Generation of CT Input Data}

Each measurement trial yields values of the integrated absorbance, $A$, as a function of time for the IR beam passing through the vapor cloud along a single path (depicted as an arrow in Fig. 2a). Representative plots of the integrated absorbance versus time for three trials, one each for elevations of 2, 3, and $4 \mathrm{~mm}$, are presented in Fig. 6 . All three trials were taken at $\mathrm{x}=0 \mathrm{~mm}$. (Measurements at $\mathrm{z}=1 \mathrm{~mm}$ are not included because a different absorbance peak was used, as discussed above.) Five distinct stages can be seen in this plot: 1) the measured absorbance is zero before the liquid drop is injected, 2) the absorbance increases rapidly while the liquid drop is injected $(\sim \mathrm{s}<\mathrm{t}<\sim 15 \mathrm{~s}), 3)$ the absorbance remains approximately steady, with only a slight change with time ( 15 s $<\mathrm{t}<\sim 100 \mathrm{~s})$, 4) the absorbance decreases after the drop has completely evaporated and as the vapor cloud dissipates ( 100 s $<\mathrm{t}<\sim 150 \mathrm{~s}$ ), and 5) the absorbance is constant at a very low value representative of the residual vapor in the sample compartment ( $\mathrm{t}>\sim 150 \mathrm{~s})$.

Previous gravimetric and schlieren imaging experiments, which were conducted under the same conditions as those presented in this paper, have shown that the hexane and 3MP drops evaporate at constant rates and in each case the vapor cloud forms nearly instantaneously around the drop and the shape of the cloud appears unchanged during the drop lifetime [25]. Under these conditions, it would be expected that the integrated absorbance would remain constant while the drop is evaporating. However, there are two factors which affect the absorbance measurements while the drop is evaporating (stage 3) and these factors can cause a slight change in the absorbance with time, as seen in Fig. 6 by the slight decrease in the absorbance with time as occurred for the measurement at $\mathrm{z}=2 \mathrm{~mm}$, and the slight increase for the measurement at $\mathrm{z}=4 \mathrm{~mm}$. As the drop evaporates, the liquid surface slowly drops while the position of the IR beam remains constant. Thus, the IR beam moves slightly away from the surface of the drop and into a less concentrated region of the vapor cloud, which explains the decreasing trend for $\mathrm{z}=2 \mathrm{~mm}$ in Fig. 6. At the same time, a small amount of background vapor gradually accumulates, which increases the measured absorbance with time. The absorbance due to the total accumulation of background vapor is shown in Fig. 6 as the flat region at time greater than $150 \mathrm{~s}$, which is the same for all three elevations. At high elevations, where the concentration gradient is low, the increasing background vapor is more influential than the lowering drop surface and the result is an increasing absorbance measurement with time, as shown by the trend for $\mathrm{z}=4 \mathrm{~mm}$ in Fig. 6 . To exclude both of these extraneous effects, the integrated absorbance at a time just after the formation of the cloud, and therefore before any significant lowering of the drop surface or accumulation of background vapor, is computed by fitting a line to the data and using this line to compute the absorbance at the end of the liquid injection. Since during the injection period the sample compartment of the FTIR is open to enable access, the first couple of absorbance measurements after the liquid has been injected are susceptible to fluctuations caused by drafts. The sample compartment is closed immediately after the liquid is injected and any external drafts quickly die out. By extrapolating back using the linear fit of the steady data, the effects of any early fluctuations in the data are mitigated. In Fig. 6, the portion of the line that is solid indicates the range of data that was used to define the fit and the portion of the line that is dotted indicates the extrapolation to the value considered to be the representative absorbance, without the extraneous influences, for the given path through the cloud. For the data given in Fig. 6, the representative absorbance values are shown by the filled data symbols and are 105.1 a.u. $\cdot \mathrm{cm}^{-1}$ at $\mathrm{z}=2 \mathrm{~mm}, 57.3$ a.u. $\cdot \mathrm{cm}^{-1}$ at $\mathrm{z}=3 \mathrm{~mm}$, and 26.8 a.u. $\cdot \mathrm{cm}^{-1}$ at $\mathrm{z}=4 \mathrm{~mm}$. For each measurement position, this procedure is repeated to yield at least three absorbance measurements.

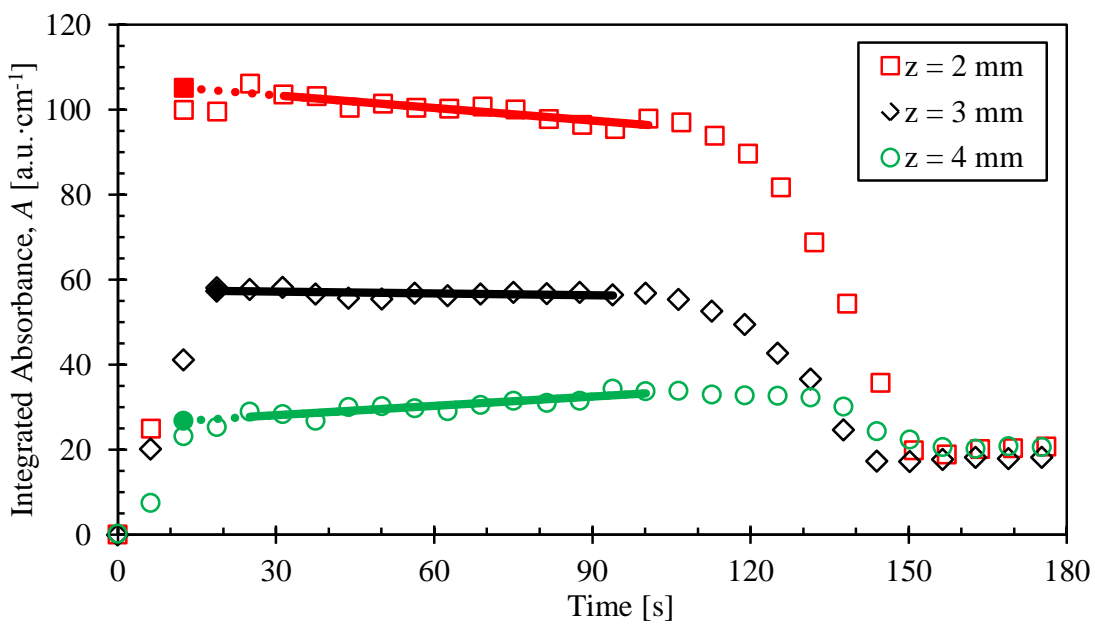

Figure 6. Representative plots of the integrated absorbance as a function of time during a measurement trial. The data was acquired for a hexane drop at $\mathrm{x}=0$ and $\mathrm{z}=2,3$, and $4 \mathrm{~mm}$.

To determine the vapor distribution in a given plane, the path-average absorbance values at multiple positions in the specified plane (the one-dimensional projection) are inputted into the CT routine. The CT routine requires a uniform interval between absorbance values of the projection, and the resolution of the resulting distribution is proportional to the resolution of the input data. Therefore, in order to determine absorbance values at equal intervals as well as to increase the resolution of the data to be inputted to the CT routine, a polynomial was fit to the measured absorbance data and the polynomial was used to compute 
absorbance values at $1 / 2 \mathrm{~mm}$ intervals. Figure 7 shows the measured absorbance as a function of position for the $\mathrm{z}=3 \mathrm{~mm}$ plane for hexane. Each datum point is the average of multiple measurements and the error bars indicate the standard deviation of the measurements. The polynomial fit to the data is also shown.

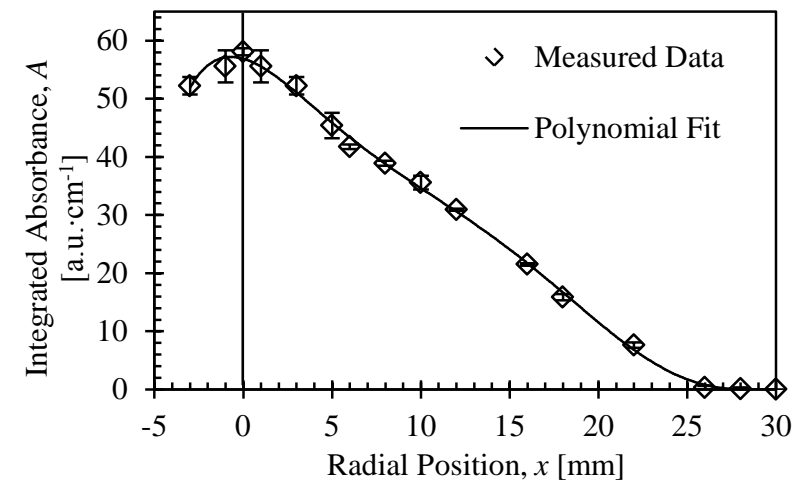

Figure 7. Distribution of integrated absorbance measurements in the $\mathrm{z}=3 \mathrm{~mm}$ plane for hexane. The data are fitted by a polynomial in order to compute absorbance values with high resolution and at a regular interval. Error bars indicate the standard deviation of the measurements at each radial position.

Since the vapor cloud is assumed to be axially symmetric, only absorbance values for $\mathrm{x} \geq 0$ are required. However, to check the symmetry assumption, near the center of the cloud some measurements were acquired for $\mathrm{x}<0$. Measurements through the full extent of the vapor cloud for $\mathrm{x}<0$ were prohibited by the dimensions of the sample compartment and the range of the translation stage. The additional measurements were used to reduce the effects of any noise in the data near the center of the cloud by averaging the measurements at $-|x|$ with those at $+|x|$ and plotting the resulting values at both $\pm|x|$. In effect, axial symmetry was used to increase the number of measurements at a given position near the center of the cloud. Extending the range of fitted data also improved the quality of the polynomial fit in the region around $\mathrm{x}=0$. Though the input to the CT routine contains only values for $\mathrm{x} \geq 0$, the routine uses symmetry to mirror the input to generate a full projection across the vapor cloud.

The elevation, $\mathrm{z}$, of the IR beam was determined as the distance from the top surface of the substrate to the geometric center of the beam. Since the half-height of the beam was $1.5 \mathrm{~mm}$ and the maximum drop thickness was about $1 \mathrm{~mm}$, a portion of the beam is unblocked at $\mathrm{z}=0$ making it possible to obtain measurements at that position. However, because much of the IR energy is blocked by the substrate and the drop, the signal-to-noise ratio was low and consequently there was a lot of measurement scatter at this position. Because of the large scatter at $\mathrm{z}=0$, measurements at that location were not used. Measurements were obtained for $\mathrm{z}=1 \mathrm{~mm}$ and greater, with a $1 \mathrm{~mm}$ increment.

\subsection{Processing the CT Results}

The output of the CT routine is a two-dimensional distribution of absorbance within a given elevation plane. This distribution was multiplied by the molar absorptivity value of the given species, which was measured as described in §2.1, to yield a twodimensional vapor concentration distribution for that plane.

The defined elevation of each of the measurement planes was raised $0.55 \mathrm{~mm}$ in order to correspond to the location of maximum intensity within the IR beam instead of the beam's geometric center. This adjustment was made because the absorbance measured by the FTIR is an intensity-weighted value and so it is influenced most by the concentration of the vapor within the portion of the beam where its intensity is highest.

After adjusting the elevations of the measured distributions, the vapor distribution between the first measured elevation plane (z $=1.55 \mathrm{~mm}$ ) and the surface of the drop was obtained by interpolation. At the surface of the drop, the vapor concentration was assumed to be saturated (at the ambient temperature) and a polynomial was fit to all of the data vertically above the given radial location. The location of the drop surface was determined from a shadowgraph image of the drop taken at a time shortly after injection. A similar procedure was used to determine the vapor concentration near the substrate surface for radii greater than the drop radius, however in this region the vertical distributions were extrapolated down to the substrate surface. 


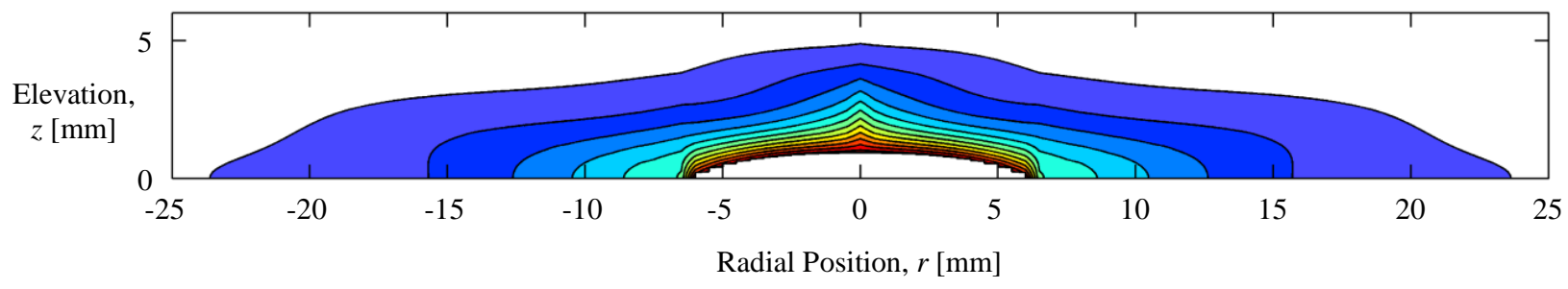

8a. Hexane vapor distribution.

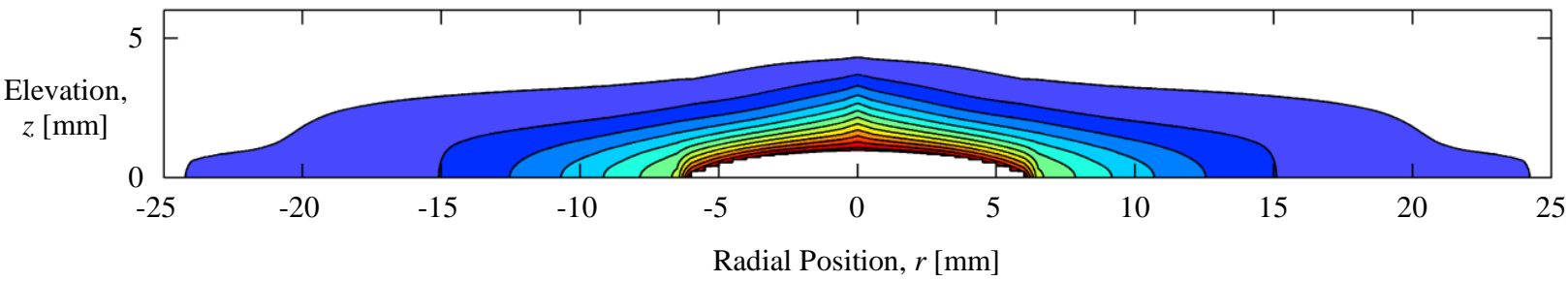

8b. 3MP vapor distribution.

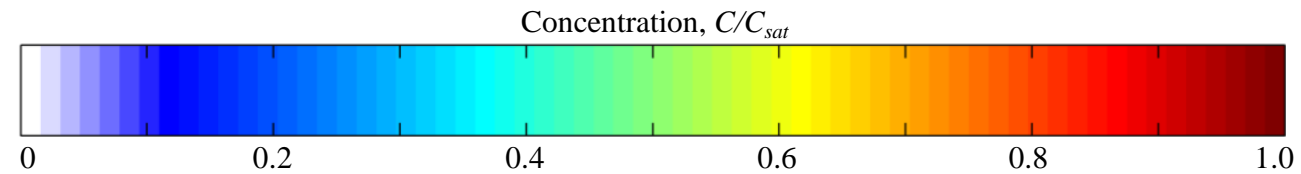

Figure 8. Computed vapor distribution for hexane (8a) and 3MP (8b) in a vertical plane passing through the center of the drop. The vapor concentration is normalized by the saturated value at $22.6^{\circ} \mathrm{C}$ and an ambient pressure of $96 \mathrm{kPa}$. The color map indicates the vapor concentration values.

\section{Results and Discussion}

Figure 8 presents the measured vapor distributions surrounding a sessile drop of hexane (8a) and 3MP (8b). Each distribution has been normalized by the saturated vapor concentration, $7.40 \times 10^{-6} \mathrm{~mol} / \mathrm{cm}^{3}$ for hexane and $8.68 \times 10^{-6} \mathrm{~mol} / \mathrm{cm}^{3}$ for $3 \mathrm{MP}$. The presented distributions are for a vertical plane passing through the center of the drop and, due to axial symmetry, they fully specify the vapor concentrations in the vapor clouds. The distributions for hexane and 3MP are nearly identical. Both of the distributions are relatively flat with a thickness of about $5 \mathrm{~mm}$ and they extend radially to about $24 \mathrm{~mm}$, which is $2 \mathrm{~mm}$ greater than the radius of the solid substrate. The geometry of these measured distributions agrees with schlieren images of the vapor clouds. For example, the schlieren image of the vapor cloud for hexane, Fig. 1, shows a flat cloud that extends radially and spills over the edge of the substrate.

A comparison of the measured vapor distribution with the distribution computed according to the assumption of diffusion-limited transport, i.e. no convective transport of the vapor, reveals significant differences. In Fig. 9 the vapor distribution computed for diffusion-limited evaporation is presented above the left half of a hexane drop and the measured vapor distribution is presented over the right half of the drop. The diffusion-limited distribution is the solution to the steady Laplace equation $[12-17,19,20,36]$. For simplicity, the solution for the drop geometry of a flat, circular hexane surface (Weber’s disk) is used [37]. This solution approximates the results for the more realistic lens-shaped drop geometry, which does not have a closed form solution [16]. For a precise quantitative comparison with the measurements, the diffusion-limited vapor distribution would have to be determined computationally for the given drop geometry.

Under conditions of diffusion-limited transport, the vapor diffuses away from the drop in a roughly hemispherical direction resulting in a distribution which extends vertically much further than the measured distribution does. In comparison, the measured distribution appears as if it has been flattened, which is compatible with the hypothesis that the heavier-than-air vapor causes a buoyancy-induced convective flow that is similar to a naturally convective flow of air over the top surface of a chilled plate. For the case of an upward facing chilled plate, the streamlines of the convective flow are directed vertically downward toward the plate and then turn horizontal along the plate surface [38]. Assuming a similar flow field around the evaporating drop, shown schematically in Fig. 9, the downward moving ambient air would push the vapor cloud down and dilute it.

An important consequence of the way in which the convective flow alters the distribution of vapor is the drastic change in the vapor concentration gradient. For the case of diffusion-limited evaporation, the concentration gradient is very low everywhere at the drop surface except around the perimeter of the drop (along the contact line). Consequently, the evaporation occurs predominately around the drop perimeter, as has been reported by multiple researchers $[15,16,19,20,36]$. In contrast, the concentration gradient for the measured distribution is comparatively high over the whole drop surface, which suggests that the evaporative flux is more evenly distributed. For example, the vertical gradient in the measured distribution $(\partial C / \partial z)$ at the surface 
of the hexane drop at $r=0$ is a factor of 4.2 greater than the gradient at the center of the liquid surface for the diffusion-limited distribution. Similarly, the radial gradient $(\partial C / \partial r)$ at the contact line is greater in the measured distribution than in the diffusionlimited distribution by a factor of 3.3. Thus not only does convection enhance drop evaporation by virtue of it being a faster transport mechanism than diffusion, also it may cause an increase in the rate of diffusion as a consequence of an increased concentration gradient. This is the nature of the coupling between convection and diffusion that is expressed in an empirical correlation for sessile drop evaporation, which was derived for a variety of heavier-than-air hydrocarbons species [39].

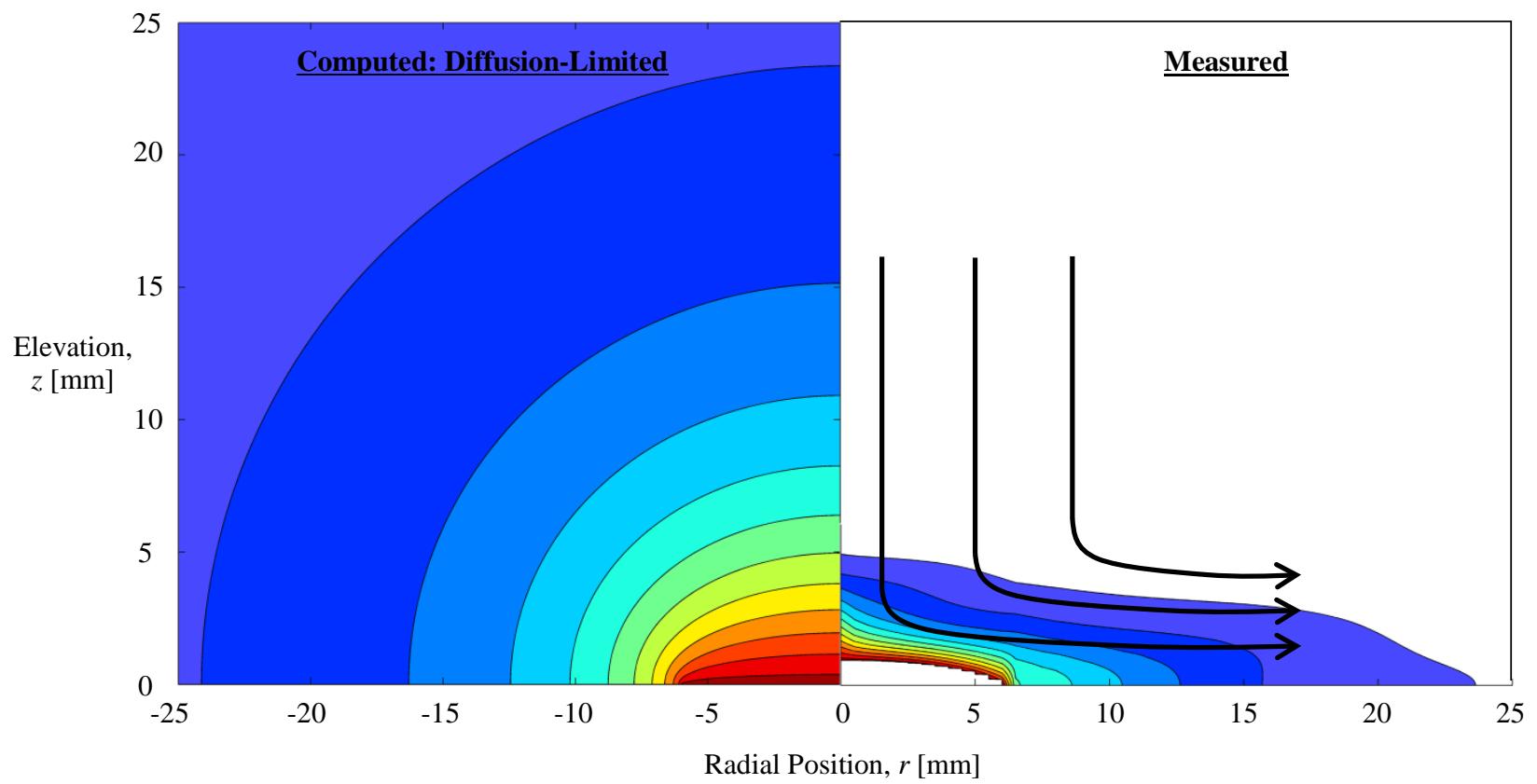

Figure 9. Comparison of vapor distributions for a hexane drop. The distribution for the condition of diffusion-limited vapor transport is given to the left of the central axis and the measured distribution is given to the right of the axis. Hypothesized streamlines of the buoyancy-induced air and vapor flow are shown by the arrows over the measured vapor distribution. Concentration values are given by the color bar in Fig. 8.

The distribution of the evaporative flux over the surface of a colloidal drop and the internal motion within the drop has been shown to affect the resulting deposition pattern [5,9,10,15,40], and thus the distribution is likely to be important for the applications of DNA stretching and depositing, and self-assembly and surface patterning. Marangoni-induced motion within a drop, which affects the resulting deposition pattern, is generated by a non-uniform surface tension distribution, which, due to evaporative cooling, is influenced by the distribution of the evaporative flux. In addition, shear stresses at the drop surface induced by the motion of the vapor-air mixture can influence the liquid motion within the drop. In this way, the motion of the drop is coupled with the vapor-phase transport mechanisms.

\section{Conclusions}

An experimental technique that combines infrared spectroscopy and computed tomography has been developed to measure the vapor concentration distribution surrounding an evaporating sessile drop. The technique was used to measure the vapor distributions surrounding drops of hexane and 3MP, and, to our knowledge, these are the first such measurements to be published.

Vapor distribution measurements are important for obtaining a thorough understanding of the transport phenomena that control sessile drop evaporation. Due to the importance of the characteristics of the vapor distribution near the drop surface in determining the nature of the evaporation process, the measurements also are important for validating computational models which are used to study a wide variety of characteristics of the evaporation process.

The measured vapor distributions of hexane and 3MP are very similar, and they both differ significantly from the distributions that would result from diffusion-limited evaporation. The differences between the measured and the diffusion-limited distributions are attributed to the effects of natural convection. It is hypothesized that the convective flow pushes the vapor down and radially away from the drop, while also diluting the vapor cloud with air. As a consequence, the concentration gradient at the drop surface is increased. Both the motion of the vapor-air mixture and the concentration gradient at the drop surface can affect the motion of the liquid drop and the distribution of the evaporative flux, two key characteristics that can influence self-assembly and surface patterning. 
Two common experimental methods are incorporated into the technique to measure the vapor distribution, Fourier transform infrared spectroscopy and computed tomography. Their use to measure the vapor distribution surrounding a sessile drop has one significant limitation. For very low elevations, less than $1 \mathrm{~mm}$ in our case, most or all of the IR beam is blocked at locations where the beam impinges on the drop. The threshold elevation depends on the diameter of the IR beam and the thickness of the drop. Because of this impingement, a complete set of absorbance measurements, which are needed for the CT routine, are not possible and consequently neither are measurements of the vapor distribution at these elevations. Instead, vapor concentration values in this region must be approximated in some way, for example by extrapolating values from the measured region.

While the technique was used to measure the vapor distribution surrounding a drop undergoing steady evaporation, the technique should be applicable also to transient evaporation if the absorbance measurements are synchronized with the evaporation process.

\section{Acknowledgements}

Acknowledgement is made to the Donors of the American Chemical Society Petroleum Research Fund for support of this research. We are also grateful to multiple students who assisted in the development of the experiments: Tim Sowers, Daoqi Wang, Alex VanTilburg, and Ryan Booth.

\section{References}

[1] W. Jia, H.-. Qiu, Experimental Investigation of Droplet Dynamics and Heat Transfer in Spray Cooling, Exp Therm Fluid Sci. 27 (2003) 829-838.

[2] K. Tio, S.S. Sadhal, Dropwise evaporation: thermal analysis of multidrop systems, Int J Heat Mass Transfer. 35 (1992) $1987-$ 2004.

[3] M. Kimura, M.J. Misner, T. Xu, S.H. Kim, T.P. Russell, Long-Range Ordering of Diblock Copolymers Induced by Droplet Pinning, Langmuir. 19 (2003) 9910-9913.

[4] B. de Gans, P.C. Duineveld, U.S. Schubert, Ink-Jet Printing of Polymers: State of the Art and Future Developments, Adv Mater. 16 (2004) 203-213.

[5] J. Park, J. Moon, Control of Colloidal Particle Deposit Patterns within Picoliter Droplets Ejected by Ink-Jet Printing, Langmuir. 22 (2006) 3506-3513.

[6] D.P. Siregar, J.G.M. Kuerten, C.W.M. van der geld, Numerical simulation of the drying of inkjet-printed droplets, J Colloid Interf Sci. 392 (2013) 388-395.

[7] M. Chopra, L. Li, H. Hu, M.A. Burns, R.G. Larson, DNA molecular configurations in an evaporating droplet near a glass surface, J Rheol. 47 (2003) 1111-1132.

[8] Y.O. Popov, Evaporative deposition patterns: Spatial dimensions of the deposit, Phys Rev E. 71 (2005) 036313-1-036313-17.

[9] T.P. Bigioni, X. Lin, T.T. Nguyen, E.I. Corwin, T.A. Witten, H.M. Jaeger, Kinetically driven self assembly of highly ordered nanoparticle monolayers, Nat Mater. 5 (2006) 265-270.

[10] R. Bhardwaj, X. Fang, D. Attinger, Pattern formation during the evaporation of a colloidal nanoliter drop: a numerical and experimental study, New J Phys. 11 (2009) 1-33.

[11] H. Nakao, S. Tokonami, T. Hamada, H. Shiigi, T. Nagaoka, F. Iwata, Y. Takeda, Direct observation of one-dimensional plasmon coupling in metallic nanofibers prepared by evaporation-induced self-assembly with DNA, Nanoscale. 4 (2012) $6814-$ 6822.

[12] N. Thomas, A. Ferguson, On Evaporation from a circular water surface, Philos Mag. 34 (1917) 309-321.

[13] R.G. Picknett, R. Bexon, The Evaporation of Sessile or Pendant Drops in Still Air, J Colloid Interf Sci. 61 (1977) $336-350$.

[14] C. Bourgès-Monnier, M.E.R. Shanahan, Influence of Evaporation on Contact Angle, Langmuir. 11 (1995) $2820-2829$. 
[15] R.D. Deegan, O. Bakajin, T.F. Dupont, G. Huber, S.R. Nagel, T.A. Witten, Contact line deposits in an evaporating drop. Phys Rev E. 62 (2000) 756-765.

[16] H. Hu, R.G. Larson, Evaporation of a Sessile Droplet on a Substrate, J Phys Chem B. 106 (2002) 1334-1344.

[17] K. Sefiane, S.K. Wilson, S. David, G.J. Dunn, B.R. Duffy, On the effect of the atmosphere on the evaporation of sessile droplets of water, Phys Fluids. 21 (2009) 062101-1-062101-9.

[18] H.Y. Erbil, Evaporation of pure liquid sessile and spherical suspended drops: A review, Adv Colloid Interfac. 170 (2012) 67-86.

[19] G.J. Dunn, S.K. Wilson, B.R. Duffy, S. David, K. Sefiane, A mathematical model for the evaporation of a thin sessile liquid droplet: Comparison between experiment and theory, Colloid Surface A. 232 (2008) 50-55.

[20] S. Semenov, V.M. Starov, R.G. Rubio, M.G. Velarde, Instantaneous distribution of fluxes in the course of evaporation of sessile liquid droplets: Computer simulations, Colloid Surface A. 372 (2010) 127-134.

[21] E. Widjaja, M.T. Harris, Numerical study of vapor phase-diffusion driven sessile drop evaporation, Comput Chem Eng. 32 (2008) 2169-2178.

[22] M.A. Saada, S. Chikh, L. Tadrist, Numerical investigation of heat and mass transfer of an evaporating sessile drop on a horizontal surface, Phys Fluids. 22 (2010) 112115-1-112115-13.

[23] M. Vynnycky, N. Maeno, Axisymmetric natural convection-driven evaporation of water: Analysis and numerical solution, Int J Heat Mass Transfer. 55 (2012) 6238-6249.

[24] R.N. O'Brien, P. Saville, Investigation of Liquid Drop Evaporation by Laser Interferometry, Langmuir. 3 (1987) 41-45.

[25] P.L. Kelly-Zion, C.J. Pursell, R.S. Booth, A.N. VanTilburg, Evaporation rates of pure hydrocarbon liquids under the influences of natural convection and diffusion, Int J Heat Mass Transfer. 52 (2009) 3305-3313.

[26] F. Carle, B. Sobac, D. Brutin, Experimental evidence of the atmospheric convective transport contribution to sessile droplet evaporation, Appl Phys Lett. 102 (2013) 061603-1-061603-4.

[27] C. Poulard, G. Guéna, A.M. Cazabat, Diffusion-driven evaporation of sessile drops, J Phys-Condens Mat. 17 (2005) S4213S4227.

[28] S.J. Carey, H. McCann, F.P. Hindle, K.B. Ozanyan, D.E. Winterbone, E. Clough, Chemical species tomography by near infra-red absorption, Chem Eng J. 77 (2000) 111-118.

[29] L. Wondraczek, A. Khorsandi, U. Willer, G. Heide, W. Schade, G.H. Frischat, Mid-infrared laser-tomographic imaging of carbon monoxide in laminar flames by difference frequency generation, Combust Flame. 138 (2004) 30-39.

[30] X. Liu, J. Liu, S. Cheong, D. Shu, J. Wang, M.W. Tate, A. Ercan, D.R. Schuette, M.J. Renzi, A. Woll, S.M. Gruner, Development of ultrafast computed tomography of highly transient fuel sprays, P Soc Photo-Opt Ins. 5535 (2004) 21-28.

[31] L.A. Todd, M. Ramanathan, K. Mottus, R. Katz, A. Dodson, G. Mihlan, Measuring chemical emissions using open-path Fourier transform infrared (OP-FTIR) spectroscopy and computer-assisted tomography, Atmos Environ. 35 (2001) 1937-1947.

[32] S. Persson, E. Oestman, The use of computed tomography in non-destructive testing of polymeric materials, aluminum and concrete: part 2 - Applications, Polym Test. 6 (1986) 415-446.

[33] A.C. Kak, M. Slaney, Principles of Computerized Tomographic Imaging, IEEE Press, New York, 1987, pp. 60.

[34] A.C. Kak, M. Slaney, Principles of Computerized Tomographic Imaging, IEEE Press, New York, 1987, pp. 186. 
[35] Matlab, Version 7.14.0.739 (R2012a), The MathWorks, Inc., Natick, Massachusetts, 2012.

[36] M. Cachile, O. Bénichou, C. Poulard, A.M. Cazabat, Evaporating Droplets, Langmuir. 18 (2002) 8070-8078.

[37] J. Crank, The Mathematics of Diffusion, 2nd ed., Oxford University Press, New York, 1975, pp. 42-43.

[38] J.V. Clifton, A.J. Chapman, Natural-convection on a finite-size horizontal plate, Int J Heat Mass Transfer. 12 (1969) 15731584.

[39] P.L. Kelly-Zion, J. Batra, C.J. Pursell, Correlation for the convective and diffusive evaporation of a sessile drop, Int J Heat Mass Transfer. 64 (2013) 278-285.

[40] H. Hu, R.G. Larson, Marangoni Effect Reverses Coffee-Ring Depositions, J Phys Chem B. 110 (2006) 7090-7094. 


\section{Table Caption}

Table 1. Molar absorptivities, integration limits, and baseline definitions for hexane and 3MP.

\section{Figure Captions}

Figure 1. Schlieren image of the vapor cloud surrounding a pinned, sessile hexane drop. The bright region indicates the presence of vapor and the arrow demonstrates a path of the infrared beam from the FTIR through the vapor cloud.

Figure 2. Schematic of a top view of the vapor cloud (grey region) and a series of IR absorption measurements made in a single elevation plane above the drop (Fig. 2a). The resulting distribution of the IR absorption measurements, which is equivalent to a one-dimensional projection of the two-dimensional absorbance distribution, is shown in Fig. $2 \mathrm{~b}$.

Figure 3. Schematic of a profile view of a sessile drop (black) which covers the surface of a disk at the center of the copper substrate. The drop is attached around the circular periphery of the disk and in this way the drop size is maintained during the majority of the evaporation process.

Figure 4. Schematic of the coordinate system centered on the top surface of the central disk. The IR beam was oriented normal to the $\mathrm{x}-\mathrm{z}$ plane.

Figure 5. Two absorbance spectra representative of measurements taken at elevations of $1 \mathrm{~mm}$ (Fig. 5a) and $3 \mathrm{~mm}$ (Fig. 5b) for IR beams passing over the center of the hexane drop. Due to the high vapor concentration directly above the drop at $\mathrm{z}=1 \mathrm{~mm}$, the absorbance peak between $3100-2800 \mathrm{~cm}^{-1}$ was saturated and so the peak between $1520-1420 \mathrm{~cm}^{-1}$ was used. For elevations greater than $1 \mathrm{~mm}$, the absorbance peak between $3100-2800 \mathrm{~cm}^{-1}$ was used. The shaded regions represent the integrated absorbance values that were used.

Figure 6. Representative plots of the integrated absorbance as a function of time during a measurement trial. The data was acquired for a hexane drop at $\mathrm{x}=0$ and $\mathrm{z}=2,3$, and $4 \mathrm{~mm}$.

Figure 7. Distribution of integrated absorbance measurements in the $\mathrm{z}=3 \mathrm{~mm}$ plane for hexane. The data are fitted by a polynomial in order to compute absorbance values with high resolution and at a regular interval. Error bars indicate the standard deviation of the measurements at each radial position.

Figure 8. Computed vapor distribution for hexane (8a) and 3MP (8b) in a vertical plane passing through the center of the drop. The vapor concentration is normalized by the saturated value at $22.6^{\circ} \mathrm{C}$ and an ambient pressure of $96 \mathrm{kPa}$. The color map indicates the vapor concentration values.

Figure 9. Comparison of vapor distributions for a hexane drop. The distribution for the condition of diffusion-limited vapor transport is given to the left of the central axis and the measured distribution is given to the right of the axis. Hypothesized streamlines of the buoyancy-induced air and vapor flow are shown by the arrows over the measured vapor distribution. Concentration values are given by the color bar in Fig. 8. 